\title{
A simple lung ultrasound protocol for the screening of COVID-19 pneumonia in the emergency department
}

\author{
Alessandro Dacrema ${ }^{1} \cdot$ Matteo Silva ${ }^{1} \cdot$ Luca Rovero $^{1}$ - Valeria Vertemati ${ }^{1}$ Giulia Losi ${ }^{1}$. Massimo Francesco Piepoli ${ }^{2}$. \\ Roberto Sacchi ${ }^{3}$. Marco Mangiacotti ${ }^{3} \cdot$ Peiman Nazerian $^{4} \cdot$ Laura Pagani $^{1} \cdot$ Valentina Tinelli $^{1} \cdot$ Erika Poggiali $^{1}$. \\ Davide Bastoni $^{1} \cdot$ Andrea Vercelli $^{1} \cdot$ Andrea Magnacavallo $^{1}$
}

Received: 9 May 2020 / Accepted: 2 December 2020 / Published online: 11 January 2021

(c) Società Italiana di Medicina Interna (SIMI) 2021

\begin{abstract}
The most relevant manifestation of coronavirus disease 2019 (COVID-19) is interstitial pneumonia. Several lung ultrasound (US) protocols for pneumonia diagnosis are used in clinical practice, but none has been proposed for COVID-19 patients' screening in the emergency department. We adopted a simplified 6-scan lung US protocol for COVID-19 pneumonia diagnosis (LUSCOP) and compared its sensitivity with high resolution computed tomography (HRCT) in patients suspected for COVID-19, presenting to one Emergency Department from February 21st to March 15th, 2020, during the outbreak burst in northern Italy. Patients were retrospectively enrolled if both LUSCOP protocol and HRCT were performed in the Emergency Department. The sensitivity of LUSCOP protocol and HRCT were compared. COVID-19 pneumonia's final diagnosis was based on real-time reverse-transcription polymerase chain reaction from nasal-pharyngeal swab and on clinical data. Out of 150 suspected COVID-19 patients, 131 were included in the study, and 130 had a final diagnosis of COVID-19 pneumonia. The most frequent lung ultrasonographic features were: bilateral B-pattern in 101 patients (77\%), B-pattern with subpleural consolidations in $26(19.8 \%)$ and lung consolidations in $2(1.5 \%)$. LUSCOP Protocol was consistent with HRCT in correctly screening 130 out of the 131 COVID-19 pneumonia cases (99.2\%). In one case COVID-19 pneumonia was excluded by both HRCT and lung US. LUSCOP protocol showed optimal sensitivity and can be proposed as a simple screening tool for COVID-19 pneumonia diagnosis in the context of outbreak burst areas where prompt isolation of suspected patients is crucial for patients' and operators' safety.
\end{abstract}

Keywords Lung ultrasound $\cdot$ COVID-19 $\cdot$ Pneumonia $\cdot$ Screening $\cdot$ Emergency $\cdot$ Outbreak

\author{
Abbreviations \\ COVID-19 Coronavirus disease 2019 \\ HRCT High resolution computed tomography \\ Lung US Lung ultrasound \\ LUSCOP Lung US for early detection of COVID-19 \\ Pneumonia
}

Andrea Magnacavallo

a.magnacavallo@ausl.pc.it

1 Emergency Department, Guglielmo Da Saliceto Hospital, via Cantone del Cristo 40, 29121 Piacenza, Italy

2 Cardiology Department, Guglielmo Da Saliceto Hospital, Piacenza, Italy

3 Earth and Environmental Sciences Department, University of Pavia, Pavia, Italy

4 Department of Emergency Medicine, Careggi University Hospital, Florence, Italy
rRT-PCR Real-time reverse-transcription polymerase chain reaction

WHO World Health Organization

\section{Introduction}

On 13th March 2020, the World Health Organization (WHO) declared the COVID-19 as a pandemic of public health concern, calling countries to activate emergency response actions [1]. The most relevant manifestation of COVID-19 is interstitial pneumonia ranging from mild to severe, i.e. acute respiratory distress syndrome (ARDS) [2]. One of the critical issues to limit COVID-19 diffusion is to have rapid and affordable screening tests. WHO is supporting the identification of a rapid point-of-care diagnostic tool [3]. In particular, this need is remarkable in the emergency departments where suspected positive patients must be isolated. 
To date, the gold standard diagnostic tool recommended for COVID-19 pneumonia is the real-time reverse transcriptionpolymerase chain reaction (rRT-PCR) to detect nucleic acid of severe acute respiratory syndrome coronavirus (SARS-CoV-2) in respiratory specimens [4]. However, a variable percentage of false-negative swabs has been documented [5]. Moreover, during the epidemic peak in highly affected areas, the long time that is required to obtain the rRT-PCR results may limit its utilization in the acute setting. Alternatively, the chest X-ray is not specific nor sensitive for the diagnosis of viral pneumonia [6]. High resolution computed tomography (HRCT) demonstrated high sensitivity and specificity for COVID-19 pneumonia, showing a characteristic pattern [7, 8]. However, HRCT has several limitations (i.e. high costing, time-consuming, radiation exposure particularly for children and pregnant women) making it not ideal for screening. Furthermore, HRCT is often unavailable in limited resource scenarios and, if available, accurate sterilization of the machine should significantly impact the diagnostic time. Recently, a more rational use of imaging tests has been suggested, reserving chest X-ray and HRCT in selected cases and assuming a role for lung US [9]. A standardized and detailed approach for lung US implementation in COVID-19 patients, based on a scanning method exploring 14 chest areas, has been recently presented, also with research purposes [10].

In the acute setting, lung US can be useful to screen suspected patients to address them to a safe path from their entrance in the emergency department: a detailed approach could be time-consuming.

In the emergency setting, lung US proved to be as accurate and reliable as HRCT and chest X-ray for the diagnosis and monitoring of viral and bacterial pneumonia [11] and ARDS [12], in both adult and children [13] and its implementation during influenza A H1N1 pandemic provided early detection of interstitial lung disease [14]. Furthermore, lung US use is widespread also in low-income countries [15].

Although the potential advantages of lung US are well known, current clinical recommendations still do not suggest a role for lung US as a screening imaging test in the emergency setting for COVID-19 pneumonia. In our real-life study, we evaluated the sensitivity of a novel, rapid, simplified lung US protocol as a screening tool in the early detection of COVID-19 pneumonia in the Emergency Department, in comparison with HRCT: the Lung US for early detection of COVID-19 Pneumonia (LUSCOP) protocol.

\section{Methods}

This was a retrospective study which considered 150 consecutive patients, with clinically suspected COVID-19, admitted to the Emergency Department of a Community Hospital in northern Italy, from February 21st to March 15th, 2020, during the COVID-19 outbreak, in one of the first and most stricken district.

Given the exponentially increasing load of patients presenting to the Emergency Department, a new allocation of resources and an adequate and safe setting for admitted patients were needed. In this context, after the triage, our Institution decided to admit suspected patients in the Emergency Department if they had moderate to severe manifestations of the disease or if they were at high risk for complications. Alternatively, patients suspected for COVID-19 with mild symptoms were evaluated in a medical station outside the Emergency Department and not included in the study because precociously discharged without second level diagnostic assessment.

The study was approved by the local Ethics Committee.

\section{Diagnostic investigations and inclusion criteria}

Patients with moderate to severe manifestation of the disease were considered those with:

1. Alterations of vital signs (blood oxygen saturation $<94 \%$, systolic blood pressure $<90 \mathrm{mmHg}$, respiratory rate $>25$ breaths/minute, heart rate $>100 \mathrm{bpm}$ )

2. Necessity for strict monitoring (i.e. patients with chest pain or syncope)

3. Alteration of the state of consciousness

4. Multimorbidity and frailty

Patients were included in the study if they met the following criteria:

1. Positive rRT-PCR from a nasal-pharyngeal swab or positive clinical diagnosis of COVID-19

2. Execution of lung US in the Emergency Department

3. Execution of HRCT in the Emergency Department

Coronavirus disease 2019 diagnostic confirmation was based on the rRT-PCR from nasal-pharyngeal swab (repeated in case of suspected false-negative results) or on the clinical diagnosis at discharge after hospitalization. Clinical diagnosis of COVID-19 was made in case of lack of swab or in case of negative rRT-PCR from nasal-pharyngeal swab but in presence of contemporary radiological confirmation, signs and symptoms and clinical course typical for COVID-19 (i.e. fever, desaturation, shortness of breath).

We adopted a rapid, simplified lung US protocol to explore posterior fields from the apex to the bases bilaterally ( 6 fields in total, 3 fields for hemithorax including apex, middle, basal field) (Fig. 1) with longitudinal scan. This choice was made on the basis of reported evidences that COVID19 pneumonia predilects the posterior part of lower lobes 
with subpleural involvement $[5,6]$. We considered lung US positive if the following were detected: B-pattern (interstitial syndrome with B-lines in clusters, both with 3 or more separate or coalescent B-lines, light beam signs, and white lung), small peripheral consolidations (subpleural echo-poor region or with tissue-like echotexture) or both findings in at least one field, as reported in the literature [16]. Lung US was a routine examination integrated into the global evaluation of the patients: after history taking and physical examination, we collected lung US data adopting a standardized form which included a scheme reporting the division of the chest in 6 posterior fields, as in Fig. 1. The forms were compiled immediately after the lung US execution and medical reports indicating the location of sonographic pathological alterations were written. We performed lung US as the first diagnostic imaging investigation, prior to performing HRCT. It was possible to perform lung US in all patients, including the critically ill, with a nurse helping the patient to maintain the proper posture if necessary. It was not possible to measure the time for lung US execution, but in expert hands, this protocol requires less than one minute.

Lung US was compared with HRCT. HRCT was considered positive on the basis of the medical radiological report, referring to the typical COVID-19 interstitial pneumonia pattern with ground-glass opacities or consolidations with peripheral subpleural distribution, mainly in the lower lobes, with or less bilateral involvement as reported in the literature $[7,8]$.

\section{Technical equipment}

Lung US was performed with different devices i.e. ESAOTE MyLab Alpha; ESAOTE Mylab 30 Gold equipped with a

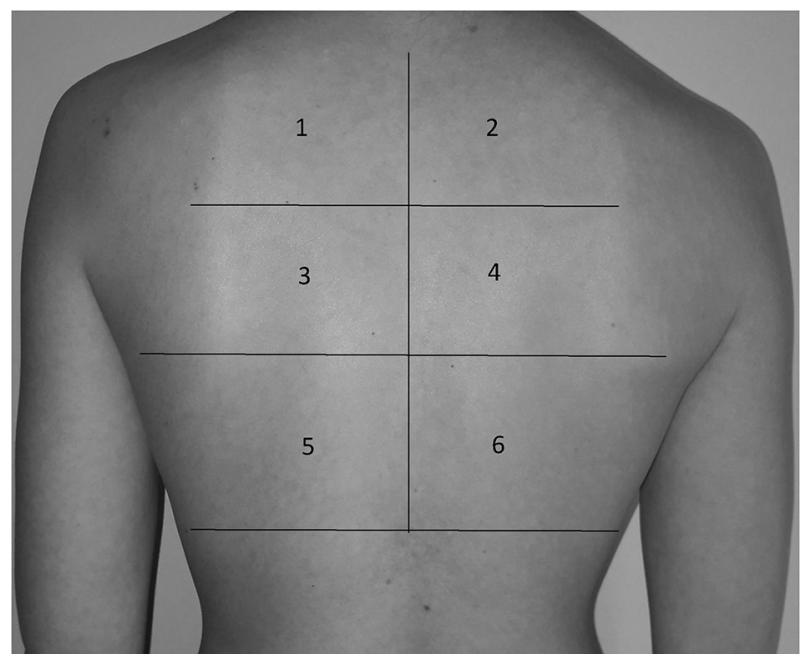

Fig. 1 Chest fields for lung US examination. Lung US scan protocol of posterior fields from the apex to the bases bilaterally: 6 fields in total, 3 fields for hemithorax, apex, middle, basal field. convex probe, probe AC2541 (frequency range 1-8 MHz), probe CA631 (frequency range $1-8 \mathrm{MHz}$ ) respectively (Esaote Medical Systems, Florence, Italy); Philips Affiniti 70 equipped with convex probe C6-2 (range frequency 2-6 MHz) (Philips N.V., Amsterdam, The Netherlands); Samsung HM70a equipped with convex probe CA1-7AD (range frequency 1-7 MHz) (Samsung Medison, Republic of Korea).

HRCT was performed with Siemens Somatom Emotion 16 Slice CT Scan Machine (Erlangen, Germany).

\section{Operators participant to the study}

The entire medical team of the Emergency Department contributed to performing lung US and the entire medical team of the Radiology Department contributed for HRCT execution.

Globally, 30 physicians performed lung US with an average of $5 \pm 3$ examinations each. Out of 30 physicians, 4 were inexperienced in lung US (less than 6 months of experience). Two of them were rapidly trained at the threshold of COVID-19 outbreak (less than 4 weeks of experience in lung US). Each operator took at least a basic course on lung US since our Community Hospital is a school of clinical ultrasound in emergency certified by SIMEU (Società Italiana di Medicina d'Emergenza-Urgenza). Overall, 6 physicians were specialists in Emergency Medicine, 10 physicians were specialists in Medical Specialties other than Emergency Medicine (Internal Medicine, Geriatrics, Sports Medicine), 14 physicians were not specialists and 11 of them received specific training for territorial health emergencies.

\section{Statistical analysis}

The reliability of lung US compared to HRCT in detecting signs suspected for COVID-19 pneumonia, was assessed by agreement analysis [17], consisting in two tests evaluating (a) the overall agreement with HRCT i.e. the rate of correctly classified cases, and (b) the occurrence of a systematic error i.e. presence of asymmetries in the wrongly classified cases. As a measure of global agreement (a), the $\Delta$ statistic was preferred to the more commonly used Cohen's $\kappa[18,19]$ because it deals with the unbalanced distribution of COVID19 pneumonia diagnosis in our cohort (130 positive vs 1 negative case, according to HRCT). Similarly to $\kappa, \Delta$ varies between -1 (complete disagreement), and +1 (complete agreement), with zero being considered a random agreement. Values above 0.8 usually indicate "almost perfect" agreement [17]. The occurrence of a systematic error was tested by the McNemar's test [20], applying Edwards' correction for continuity [21] and a binomial exact test, given the low frequency of the wrongly classified cases. All the 
analyses were run in the $\mathrm{R}$ environment [22] using basic functions and the 'Delta' package [23].

\section{Results}

\section{Patients enrollment}

Out of the 150 consecutive recruited patients, 19 were excluded because they did not meet the inclusion criteria (Fig. 2). Only those with confirmed COVID-19 diagnosis and both lung US and HRCT medical reports were enrolled. Hence 131 patients were finally considered for statistical analysis. The sample population included 32 women (24.4\%) and 99 men $(75.6 \%)$ between 19 and 94 years of age $($ mean \pm SD: $64.3 \pm 14.3)$. Out of 131 enrolled patients, 124 were hospitalized, 7 were discharged from the Emergency Department.

\section{Patients' characteristics and lung ultrasonographic features on admission}

On admission to the Emergency Department, the majority of patients (69.5\%) suffered from shortness of breath, with symptoms starting on average $7 \pm 3$ days before. The $15.3 \%$ of individuals had a positive medical history for lung disease and the $\mathrm{PaO}_{2} / \mathrm{FiO}_{2}$ assessed on admission ranged from 50 to 500 , being on average $249 \pm 91$. In our cohort patients with atypical manifestations like syncope/pre-syncope $(n=6)$, chest pain $(n=2)$, and trauma $(n=1)$ were included (Table 1).

The most frequent lung ultrasonographic features were: bilateral B-pattern (including B-lines in clusters with 3 or more separate or coalescent B-lines, light beam signs, and white lung) in $77.1 \%$ of patients $(n=101)$, B-pattern with subpleural consolidations in $19.8 \%$ of patients $(n=26)$ and lung consolidations in $1.5 \%$ of patients $(n=2)$ (Table 1$)$.

\section{LUSCOP protocol sensitivity}

Out of the 131 COVID-19 enrolled patients, the LUSCOP protocol was consistent with HRCT in classifying 130 diagnoses $(99.2 \%$ ) (Figs. 3, 4), corresponding to $\Delta=0.9666 \pm 0.0204$ (mean \pm standard error). One case of COVID-19 pneumonia was not identified by lung US. The classification of error excluded the occurrence of a systematic error of lung US compared to HRCT, considering HRCT as the gold standard to assess reliability of lung

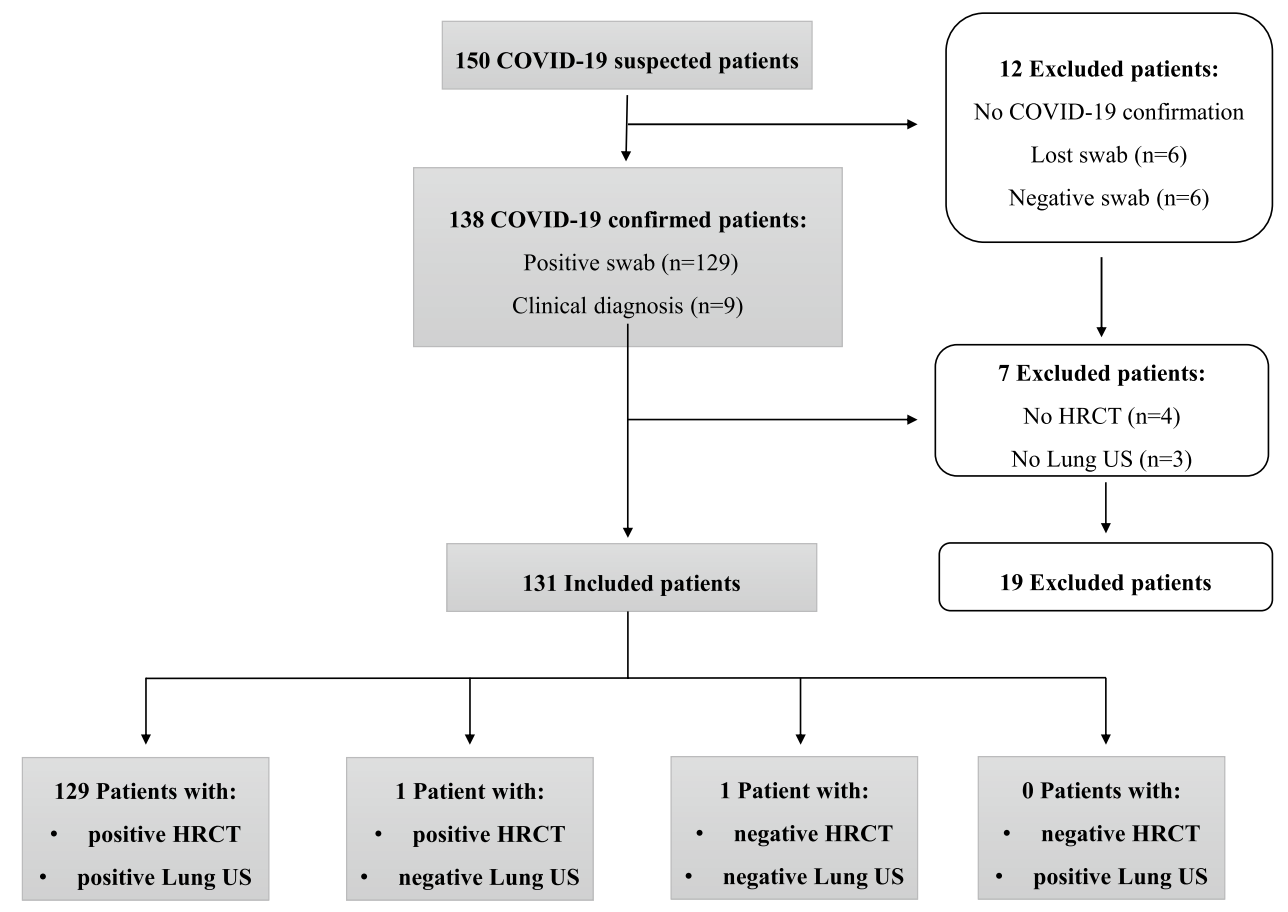

Fig. 2 Flowchart of patients' enrollment. Hundred-fifty consecutive patients suspected for COVID-19 were recruited. For 6 patients, nasal-pharyngeal swab was lost and 6 patients had a negative result, therefore 12 patients had not COVID-19 confirmed diagnosis and were excluded. Of the 138 remaining patients, 129 patients had positive rRT-PCR from nasal-pharyngeal swab, and 9 had clinical diagnosis of COVID-19. Of the 129 patients, 5 patients had a false negative result from the first swab and a subsequent diagnostic confirmation from the second swab. Therefore, 138 patients had COVID-19 diagnostic confirmation. Of the 138 patients, 4 were excluded because HRCT was not performed and 3 because lung US was either not performed or not recorded on the medical file. Finally, 19 patients were excluded and 131 patients with confirmed COVID-19 diagnosis that underwent both lung US and HRCT were enrolled. 
Table 1 Patients characteristics and lung ultrasonographic features on admission

\begin{tabular}{|c|c|c|c|}
\hline & Women $n=32$ & Men $n=99$ & Whole sample $n=131$ \\
\hline Age & $\begin{array}{l}67.9 \pm 14.3 \\
(33-94)\end{array}$ & $\begin{array}{l}63.1 \pm 14.2 \\
(19-91)\end{array}$ & $\begin{array}{l}64.3 \pm 14.3 \\
(19-94)\end{array}$ \\
\hline Temperature $\left({ }^{\circ} \mathrm{C}\right)$ & $\begin{array}{l}37.8 \pm 1.0 \\
(36-39.6)\end{array}$ & $\begin{array}{l}37.7 \pm 1.0 \\
(35-40)\end{array}$ & $\begin{array}{l}37.7 \pm 1.0 \\
(35-40)\end{array}$ \\
\hline Duration of symptoms (days) & $\begin{array}{l}6.1 \pm 2.9 \\
(0-11)\end{array}$ & $\begin{array}{l}7.5 \pm 3.3 \\
(0-19)\end{array}$ & $\begin{array}{l}7.1 \pm 3.3 \\
(0-19)\end{array}$ \\
\hline $\mathrm{PaO}_{2} / \mathrm{FiO}_{2}$ & $\begin{array}{l}246.1 \pm 99.2 \\
(50-438)\end{array}$ & $\begin{array}{l}250.5 \pm 89.3 \\
(50-500)\end{array}$ & $\begin{array}{l}249.4 \pm 91.4 \\
(50-500)\end{array}$ \\
\hline \multicolumn{4}{|l|}{ Main symptoms ${ }^{\mathrm{a}}$} \\
\hline Flu-like symptoms & $6(18.7 \%)$ & $25(25.2 \%)$ & $31(23.7 \%)$ \\
\hline Shortness of breath & $24(75.0 \%)$ & $67(67.7 \%)$ & $91(69.5 \%)$ \\
\hline Syncope/pre-syncope & $1(3.1 \%)$ & $5(5.1 \%)$ & $6(4.6 \%)$ \\
\hline Trauma & 0 & $1(1.0 \%)$ & $1(0.7 \%)$ \\
\hline Chest pain & $1(3.1 \%)$ & $1(1.0 \%)$ & $2(1.5 \%)$ \\
\hline \multicolumn{4}{|l|}{ Lung disease } \\
\hline Yes & $8(25.0 \%)$ & $12(12.1 \%)$ & $20(15.3 \%)$ \\
\hline No & $24(75.0 \%)$ & $87(87.9 \%)$ & $111(84.7 \%)$ \\
\hline \multicolumn{4}{|l|}{ Comorbidities $^{\mathrm{b}}$} \\
\hline 0 & $8(25.0 \%)$ & $41(41.4 \%)$ & $49(37.4 \%)$ \\
\hline 1 & $7(21.9 \%)$ & $14(14.1 \%)$ & $21(16.0 \%)$ \\
\hline 2 & $17(53.1 \%)$ & $44(44.5 \%)$ & $61(45.6 \%)$ \\
\hline \multicolumn{4}{|l|}{ Lung US features } \\
\hline Positive (bilateral B-pattern) & $21(65.6 \%)$ & $80(80.8 \%)$ & $101(77.1 \%)$ \\
\hline $\begin{array}{l}\text { Positive (bilateral B pattern }+ \text { con- } \\
\text { solidations) }\end{array}$ & $10(31.2 \%)$ & $16(16.2 \%)$ & $26(19.8 \%)$ \\
\hline Positive (consolidations) & 0 & $2(2.0 \%)$ & $2(1.5 \%)$ \\
\hline Negative & 0 & $1(1.0 \%)$ & $1(0.8 \%)$ \\
\hline Negative (pleural effusion) & $1(3.1 \%)$ & 0 & $1(0.8 \%)$ \\
\hline \multicolumn{4}{|l|}{ Hospitalized patients } \\
\hline Yes & $31(96.9 \%)$ & $93(93.9 \%)$ & $124(94.7 \%)$ \\
\hline No & $\begin{array}{l}1 \\
(3.1 \%)\end{array}$ & $\begin{array}{l}6 \\
(6.1 \%)\end{array}$ & $\begin{array}{l}7 \\
(5.3 \%)\end{array}$ \\
\hline
\end{tabular}

Reported values are means \pm standard deviations and ranges of variation

${ }^{a}$ Main symptoms refers to the main symptom that led the patients to the Emergency Department

${ }^{\mathrm{b}}$ Comorbities: $0=$ no comorbidities; $1=$ one comorbidity, including known lung disease; $2=$ two or more comorbidities
US (McNemar test $d^{2}=0.00 ; P>0.05$; binomial exact test $P>0.05)$. In 129 patients, COVID-19 pneumonia was confirmed by both HRCT and lung US. In two cases lung US was considered negative. In one of them, COVID-19 pneumonia was excluded by both HRCT and lung US despite the positivity of rRT-PCR from nasal-pharyngeal swab (Table 2). In this particular case, both HRCT and lung the US failed to detect signs of COVID-19 pneumonia in the presence of significant bilateral pleural effusion: failure could be attributed to pulmonary atelectasis and signs of interstitial pneumonia could have been hidden.

\section{Discussion}

Lung US for early detection of COVID-19 Pneumonia (LUSCOP) protocol is a reliable method for COVID-19 pneumonia screening in comparison to HRCT in a reallife scenario in the Emergency Department. Despite the simplified and rapid approach, the high sensitivity of the LUSCOP protocol is explainable because of the typical COVID-19 interstitial pneumonia pattern that has a peripheral, posterior distribution with subpleural 

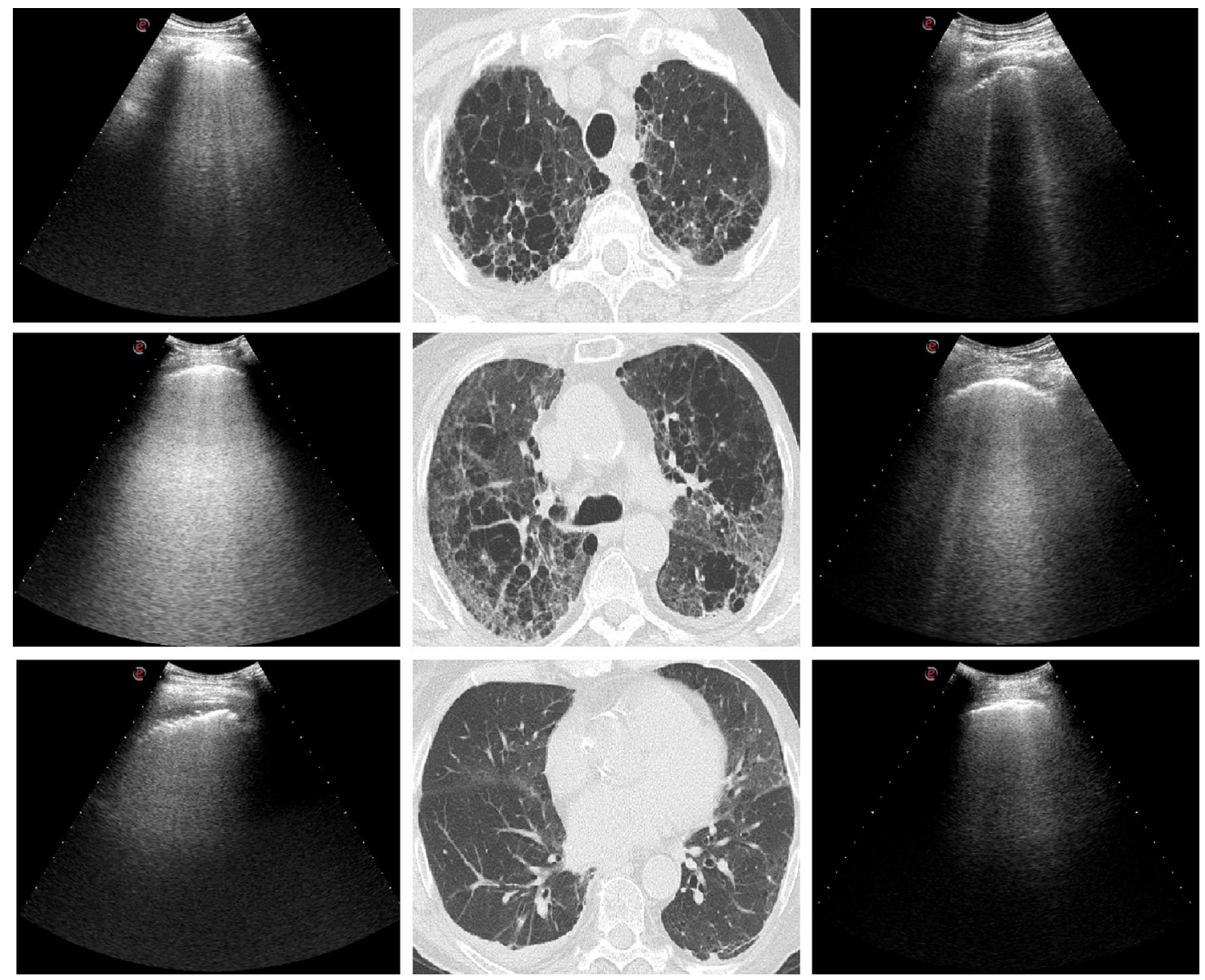

Fig. 3 Comparison between HRCT and lung US according to LUSCOP protocol. From top to bottom: upper, medium, and lower fields are shown both in HRCT slices and in the corresponding right and left lung US. HRCT shows diffuse pulmonary emphysema mostly in upper and medium fields, associated with COVID-19 bilateral dorsal, subpleural ground-glass opacities, and consolidations. In

involvement, making it easily detectable by this imaging technique.

In our real-life study, performed during the COVID-19 outbreak burst, LUSCOP protocol showed a few interesting features:

1. Its sensitivity in COVID-19 pneumonia screening seemed not to be influenced by the rapid simplified 6 fields approach in patients with moderate to severe manifestations of the disease

2. It detected signs of pneumonia also in patients complaining of atypical symptoms

3. It was feasible because performed by many operators with reliable results and implemented efficiently on patients with severe manifestations of the disease, too

4. Its diagnostic sensitivity was high also considering inexperienced operators the upper fields, B-lines at the lung US correspond to areas of mild interstitial involvement. In the middle fields, lung ultrasound shows confluent B-lines with pleural thickening. In the lower fields, pleural effusion is detected by lung US in the right side, associated with pleural thickening and irregularity; the left lung US shows a predominantly A-pattern corresponding to relatively spared parenchyma.

A role of lung US for early diagnosis of COVID-19 pneumonia has been hypothesized [24].

Lack of sensitivity of nasal-pharyngeal swab for COVID-19 pneumonia in comparison with HRCT has been demonstrated [25]. In our experience, rRT-PCR from nasal-pharyngeal swab took hours to days to be processed because of the laboratory overload. Moreover, there were a few false-negative swabs at the first sample and with a few of lost results (Fig. 2).

In our cohort, we used lung US in suspected patients as the first imaging approach, with high sensitivity for COVID-19 pneumonia detection in comparison with HRCT.

Lung US can be easily implemented while waiting for molecular diagnostic confirmation with rRT-PCR from a nasal-pharyngeal swab or for possible radiological confirmation if indicated, with many advantages. 

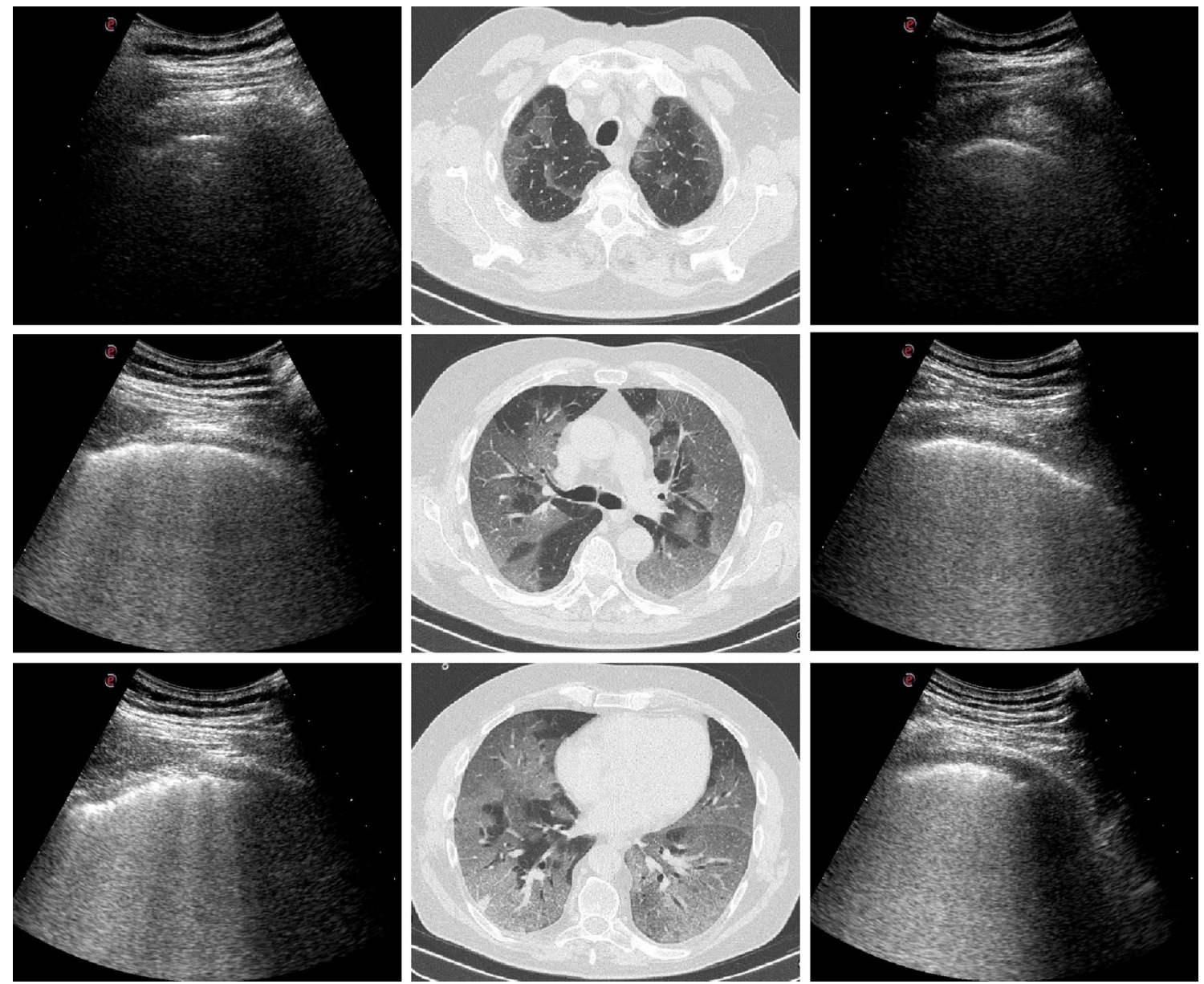

Fig. 4 Comparison between HRCT and lung US according to LUSCOP protocol. From top to bottom: upper, medium, and lower fields are shown both in HRCT slices and in the corresponding right and left lung US. In this case, HRCT shows extensive COVID-19 pneumonia involving both lungs, with subpleural involvement, partially sparing the anterior and upper fields. Specifically, crazy paving

Table 2 Confusion matrix comparing the frequency of positive and negative diagnoses according to lung US (rows) and HRCT (columns)

\begin{tabular}{lll}
\hline Lung US & $\begin{array}{l}\text { HRCT (Standard) } \\
\text { Positive }\end{array}$ & Negative \\
\hline Positive & 129 & 0 \\
Negative & 1 & 1 \\
\hline
\end{tabular}

Lung US lung ultrasound, HRCT high resolution computed tomography

First of all, initial screening with lung US allows to perform a bedside evaluation and rapidly isolate positive patients. Indeed, a prompt isolation could avoid the spread of the virus during patients' stay in the emergency department and minimize patients' and operators' risks. Many lung US scan protocols are available but, in the state pattern superimposition on ground glass opacities had an incremental cranio-caudal distribution with higher density in the subpleural dorsal fields. In the upper fields, lung US shows spared parenchyma. In the medium and lower fields, lung US shows an increasing B-pattern severity with confluent $\mathrm{B}$ lines and progressive pleural thickening with pre-consolidative state.

of necessity of COVID-19 outbreak burst, we adopted a rapid, simplified posterior scan protocol, with proven efficacy on the basis of typical HRCT radiologic features [6-8]. This approach permitted a rapid isolation of suspected patients with a quick look method. Working in an epidemic and emergency setting did not allow to perform a whole lung US with anterior, lateral, and posterior thoracic scan detection that can be reserved for a detailed second evaluation.

Furthermore, lung US for the detection of B-pattern is rapid and reliable also in rapidly trained operators [26], and can be easily implemented in clinical practice, also in centers or by operators not used to lung US employment.

Lung US can avoid chest X-ray or HRCT for COVID-19 pneumonia reserving their implementation in selected cases, according to the Multinational Consensus Statement from the Fleischner Society [9]. 
With this approach, the contamination risk and the costs in terms of time, human resources commitment, and finances would be limited and rationalized.

This rapid, simplified, lung US protocol has been effectively used in the state of COVID-19 pandemic emergency for the screening of suspected patients. After this experience, we decided to adopt a post triage lung US station for screening evaluation of suspected patients, to address them to the most proper area in the Emergency Department.

\section{Limitations}

This is a retrospective study performed in a state of emergency. Because of the high prevalence of COVID-19, an overestimation of diagnostic sensitivity of lung US for COVID-19 pneumonia is possible, and bias related to the retrospective evaluation should be present.

Limitations of this study include the absence of data about the statistical specificity of the LUSCOP protocol. Furthermore, the LUSCOP protocol does not identify specific signs of COVID-19 pneumonia. Because of the inclusion criteria, its diagnostic accuracy in patients with mild manifestations of COVID-19 has not been verified. Despite the LUSCOP protocol suggests a short execution time, we did not have the opportunity to collect precise data about the mean duration of the procedure.

\section{Conclusions}

In the context of COVID-19 outbreak burst, a novel, simple lung US protocol (LUSCOP protocol) showed to be a feasible, reliable, and sensitive screening tool for COVID-19 interstitial pneumonia diagnosis in patients with moderate/ severe manifestations of the disease.

Acknowledgements A special thanks to the hospital staff members, in particular to the Emergency Department for the efforts made during these hard days, with particular thought to the patients, healthcare personnel, and their families.

Author contributions AM was the creator of lung US screening method and responsible for its application in Emergency Department; AD prepared the study design and protocol and submitted the protocol to the Ethical Committee; AD had full access to the data, was responsible for data integrity, analysis, and critical data interpretation; AD, MFP, PN were responsible for article writing, MFP and PN were responsible for methodological quality assessment and critical revision of the article; LR, VV, and GL were responsible for data collection and database implementation and compilation; DB created the first database version; RS and MM were responsible for statistical analysis and data interpretation; LP, VT, GL, EP, and DB were clinical data collectors; AV and MS were team coordinator for clinical data collection and database compilation; AM was the team leader of the study and final reviewer of the manuscript. All authors have read, revised, and approved the manuscript.
Funding None.

Data availability Not applicable.

\section{Compliance with ethical standards}

Conflicts of interest The authors declare that they have no conflict of interest.

Ethics approval The local Ethics Committee "Comitato Etico AVEN dell'Area Vasta Emilia Nord" approved the study.

Informed consent Informed Consent was waived because of the retrospective nature of the study. All the procedures and data were part of routine care.

\section{References}

1. https://www.who.int/emergencies/diseases/novel-coronaviru s-2019/events-as-they-happen. Accessed April 20, 2020.

2. Guan WJ, Ni ZY, Hu Y et al (2020) Clinical characteristics of coronavirus disease 2019 in China. N Engl J Med. https://doi. org/10.1056/NEJMoa2002032

3. Coronavirus disease (COVID-19) situation reports. Situation report 25. 14 February 2020. http://who.int/emergencies/disea ses/novel-coronavirus-2019/situation-reports. Accessed April 20, 2020.

4. Chinese clinical guidance for COVID-19 pneumonia diagnosis and treatment (7th edition). March 2020. http://kjfy.meetingchi na.org/msite/news/show/cn/3337.html. Accessed April 20, 2020.

5. Fang Y, Zhang H, Xie J, Lin M, Ying L, Pang P, Ji W (2020) Sensitivity of chest CT for COVID-19: comparison to RT-PCR. Radiology 19:200432

6. Zu ZY, Jiang MD, Xu PP, Chen W, Ni QQ, Lu GM, Zhang LJ (2020) Coronavirus disease 2019 (COVID-19): a perspective from China. Radiology 2020:200490

7. Salehi S, Abedi A, Balakrishnan S (2020) Gholamrezanezhad A (2020) Coronavirus disease 2019 (COVID-19): a systematic review of imaging findings in 919 patients. Am J Roentgenol $14: 1-7$

8. Koo HJ, Lim S, Choe J, Choi SH, Sung H, Do KH (2018) Radiographic and CT features of viral pneumonia. Radiographics 38:719-739

9. Rubin GD, Ryerson CJ, Haramati LB et al (2020) The role of chest imaging in patient management during the COVID-19 pandemic: a multinational consensus statement from the Fleischner society. Radiology. https://doi.org/10.1148/radiol.2020201365

10. Soldati G, Smargiassi A, Inchingolo R et al (2020) Proposal for international standardization of the use of lung ultrasound for patients With COVID-19: a simple quantitative, reproducible method. J Ultrasound Med. https://doi.org/10.1002/jum.15285

11. Nazerian P, Volpicelli G, Vanni S et al (2015) Accuracy of lung ultrasound for the diagnosis of consolidations when compared to chest computed tomography. Am J Emerg Med 33(5):620-625

12. Mongodi S, Bonaiti S, Stella A, Bouhemad B, Stella A, Tavazzi G, Via G, Iotti GA, Mojoli F (2019) Lung ultrasound for daily monitoring and management of ARDS patients. Clinic Pulm Med 26(3):92-97

13. Berce V, Tomazin M, Gorenjak M, Berce T, Lovrenčič B (2019) The usefulness of lung ultrasound for the aetiological diagnosis of community-acquired pneumonia in children. Sci Rep 9(1):17957 
14. Testa A, Soldati G, Copetti R, Giannuzzi R, Portale G, GentiloniSilveri N (2012) Early recognition of the 2009 pandemic influenza A (H1N1) pneumonia by chest ultrasound. Crit Care 16(1):R30

15. Amatya Y, Rupp J, Russell FM, Saunders J, Bales B, House DR (2018) Diagnostic use of lung ultrasound compared to chest radiograph for suspected pneumonia in a resource-limited setting. Int $\mathbf{J}$ Emerg Med 11(1):8

16. Volpicelli G, Gargani L (2020) Sonographic signs and patterns of COVID-19 pneumonia. Ultrasound J 12(1):22

17. Watson PF, Petrie A (2010) Method agreement analysis: a review of correct methodology. Theriogenology 73(9):1167-1179

18. Nelson JC, Pepe MS (2000) Statistical description of interrater variability in ordinal ratings. Stat Methods Med Res 9(5):475-496

19. Andrés AM, Marzo PF (2004) Delta: a new measure of agreement between two raters. Br J Math Stat Psychol 57(Pt 1):1-19

20. McNemar Q (1947) Note on the sampling error of the difference between correlated proportions or percentages. Psychometrika 12(2):153-157

21. Edwards AL (1948) Note on the correction for continuity in testing the significance of the difference between correlated proportions. Psychometrika 13(3):185-187

22. R Core Team (2018) R: A language and environment for statistical computing. R Foundation for Statistical Computing, Vienna, Austria. https://www.R-project.org/.
23. Rodriguez A (2019) Delta: measure of agreement between two raters. R package version 0.2.0.3. https://CRAN.R-project.org/ package $=$ Delta

24. Poggiali E, Dacrema A, Bastoni D et al (2020) Can lung US help critical care clinicians in the early diagnosis of novel coronavirus (COVID-19) pneumonia? Radiology 13:200847

25. Ai T, Yang Z, Hou H, Zhan C, Chen C, Lv W, Tao Q, Sun Z, Xia $\mathrm{L}$ (2020) Correlation of chest CT and RT-PCR testing in coronavirus disease 2019 (COVID-19) in China: a report of 1014 cases. Radiology 26:200642

26. Volpicelli G, Elbarbary M, Blaivas M et al (2012) International evidence-based recommendations for point-of-care lung ultrasound consensus conference on lung ultrasound (ICC-LUS). Intensive Care Med 38(3):577-591

Publisher's Note Springer Nature remains neutral with regard to jurisdictional claims in published maps and institutional affiliations. 